\title{
The course of pregnancy and delivery in a patient with malaria
}

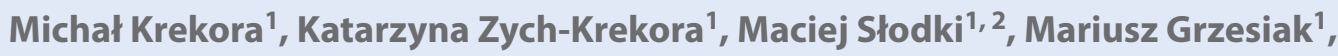 \\ Maria Respondek-Liberska ${ }^{1}$ \\ ${ }^{1}$ Mother's Memorial Hospital Research Institute in Łódź, Poland \\ ${ }^{2}$ The State University of Applied Sciences in Płock, Poland
}

Malaria is one of the most common lethal parasitic diseases. Infection is transmitted when an infected female mosquito bites a human introducing the sporozoites into human blood.

The article presents the course of pregnancy and delivery in a patient complicated by Plasmodium infection. The patient had repetitive several trips to Tanzania over a short time period before she developed the condition. She had been taking antimalarial medication (proguanil-atovaquone) in a prophylactic dose during and after her first travel to Tanzania. Following her first return to Poland she experienced infection-related symptoms.

During her second trip to Tanzania the patient continued to receive the prophylactic dose of antimalarial drug. However, due to missed menstruation and a suspicion of pregnancy, the patient has discontinued the drug without prior medical consultation since she was not certain what possible effects the drug may have on the embryo. Live pregnancy was confirmed by an ultrasound scan. As the third trip was approaching the patient decided not to take antimalarial drugs and simply avoid leaving the worksite and the hotel room as malaria prevention measures while abroad. However, halfway through her trip the patient experienced nausea and vomiting. Initially, the patient was unsuspicious since according to her knowledge such symptoms were natural during the initial stage of pregnancy. However, due to progressing anorexia with declining well-being and the onset of osteoarticular pains interfering with her work and everyday activities, the patient has decided to report at a local doctor's office (Fig. 1). Based on the intensity of symptoms the local physician has referred the patient for a rapid diagnostic test for malaria infection (Fig. 2). The result of the QBC Malaria Test was positive. Upon the diagnosis of acute infection antimalarial treatment was initiated with artemether. After the completion of the treatment and the return to Poland another malaria screening test was performed, this time yielding a negative result. Simultaneously, due to slight anemia in the course of Plasmodium infection, a decision was made to initiate administration of folic acid (5 mg). No deviations from normal values were observed over the successive weeks, neither in biochemical assays nor in ultrasound scans. At the $36^{\text {th }}$ week of gestation the patient was diagnosed with cholestasis on the basis of clinical presentation and a high level of biochemical markers. Oral treatment was initiated (ursodeoxycholic acid). Due to the abnormal CTG record exhibiting decelerations and the increasing hepatic parameters the patient was referred to the hospital where she was qualified for labor induction. The patient gave birth to a male neonate weighing $3860 \mathrm{~g}$, Apgar score 9/10.

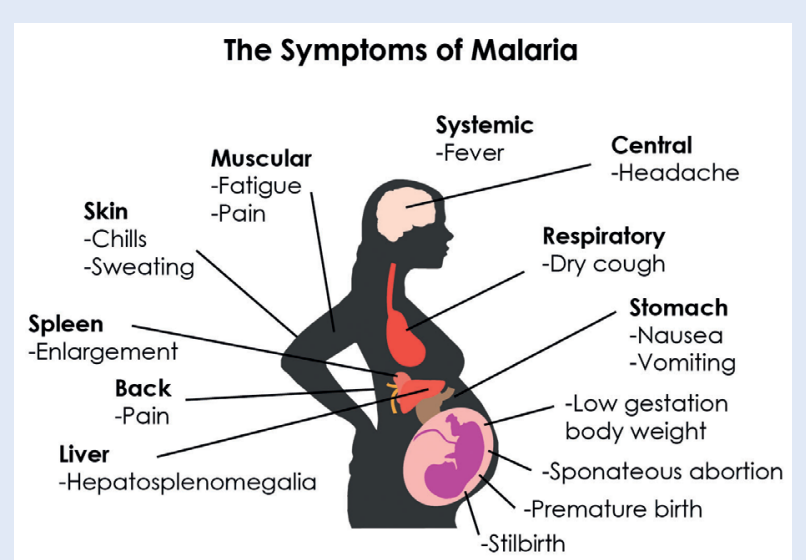

Figure 1. The symptoms of malaria 
About 125 million pregnant women are at risk of malaria infection each year.

Despite the fact that malaria is encountered mainly in Africa, one should be aware that Europe is also at risk due to changes in climate as well as massive migrations of populations, particularly in recent times. Invasive mosquitoes capable of transmitting the infectious parasite were detected in Germany and also in Slovakia.

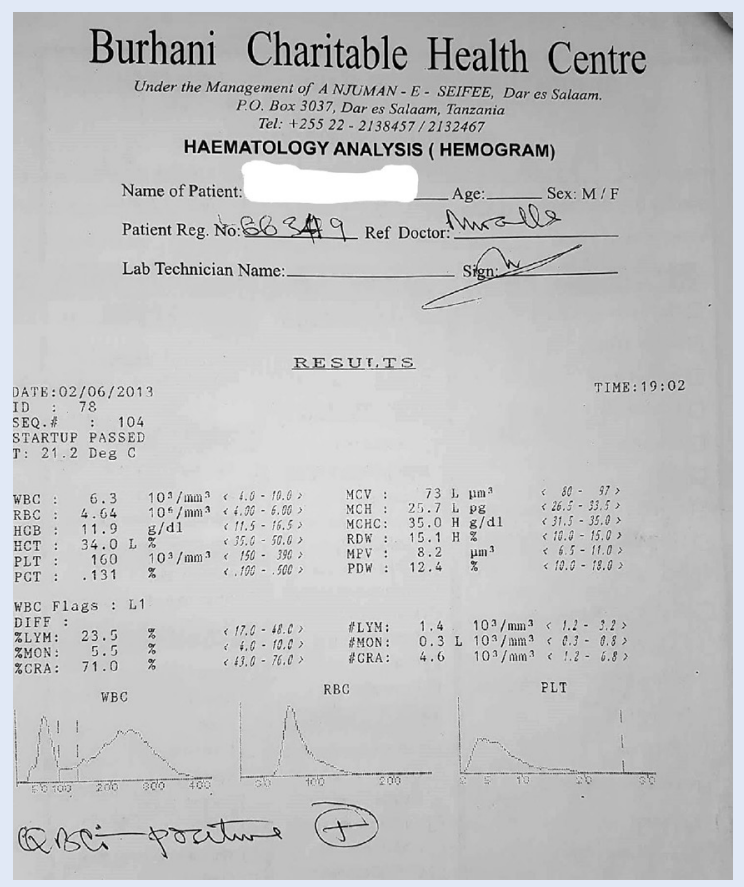

Figure 2. Blood results from Tanzania 